\title{
Modelo para estimativa da geração de resíduos sólidos domiciliares em centros urbanos a partir de variáveis socioeconômicas conjunturais
}

\section{Model to domestic solid waste generation estimative in urban areas based on socioeconomic conjuncture variables}

\author{
David Montero Dias \\ Doutor em Saneamento, Meio Ambiente e Recursos Hídricos pela Universidade Federal de Minas Gerais (UFMG). Analista de Planejamento do Instituto \\ Brasileiro de Geografia e Estatística (IBGE) - São Paulo (SP), Brasil.
}

\section{Carlos Barreira Martinez}

Doutor em Planejamento Energético pela Universidade Estadual de Campinas (UNICAMP). Professor Associado do Departamento de Engenharia Hidráulica e Recursos Hídricos da UFMG - Belo Horizonte (MG), Brasil.

\section{Raphael Tobias Vasconcelos Barros}

Doutor em Engenharia Sanitária pelo Institut National des Sciences Apliquées de Lyon. Professor Adjunto do Departamento de Engenharia Sanitária e Ambiental da UFMG - Belo Horizonte (MG), Brasil.

\section{Marcelo Libânio}

Professor Associado do Departamento de Engenharia Hidráulica e Recursos Hídricos da UFMG - Belo Horizonte (MG), Brasil.

\section{Resumo}

Este trabalho apresenta um modelo matemático para estimar a geração de resíduos sólidos residenciais urbanos. O modelo foi desenvolvido a partir de uma base física espacial de bairros de Belo Horizonte e dos indicadores socioeconômicos das respectivas populações, referentes ao período de janeiro de 2006 a dezembro de 2010. Verificou-se intrínseca relação entre a geração per capita de resíduos e a renda per capita nas regiões estudadas, testificada por coeficiente de determinação de 0,85. Posteriormente, efetuou-se a validação do modelo com dados da capital de Minas Gerais e de Porto Alegre, na qual os valores estimados foram confrontados com a geração real informada pelas prestadoras de serviços de limpeza urbana locais. A despeito de elaborado com dados de Belo Horizonte e aferido com os de Porto Alegre, os resultados abrem a perspectiva de emprego do modelo proposto na estimativa de geração de resíduos sólidos em áreas urbanas.

Palavras-chave: resíduos sólidos urbanos; geração de resíduos sólidos; renda per capita; modelo de geração de resíduos sólidos.

\begin{abstract}
This work presents a mathematical model to estimate the solid waste generation in urban areas. The model was developed based on spatial physic basis of neighborhoods of Belo Horizonte and of the respective economic indicators of their population. A strong relation between per capita solid waste generation and per capita income was confirmed due the high coefficient of the determination 0.85. Afterwards the model was validated by means of Belo Horizonte and Porto Alegre datasets in which the estimated values were compared with the real solid waste generation provided by the local services. Despite of data set from Belo Horizonte, the results open the perspective of the application of the model for estimating the solid waste generation in urban areas.
\end{abstract}

Keywords: urban solid waste; solid waste generation; per capita income; model for solid waste generation. 


\section{Introdução}

Atualmente, a gestão dos resíduos sólidos urbanos (RSU) consolida-se como uma das muitas questões ambientais prementes do mundo contemporâneo. A especificidade e complexidade dos resíduos domiciliares remetem à constante produção por indivíduo, família ou domicílio. No tocante à classificação dos resíduos sólidos, de forma complementar à estabelecida pela NBR 10004 (ABNT, 2004), a Lei Nacional de Resíduos Sólidos (12.305/2010), em seu artigo 3º, classifica-os quanto à origem como residenciais, comerciais, públicos, domiciliares especiais (entulhos de obras, pilhas e baterias, lâmpadas fluorescentes, pneus) e de fontes especiais, tais como industriais, radioativos, de portos, aeroportos e terminais rodoviários, agrícolas, de saúde e domiciliares em cidades turísticas.

O desenvolvimento econômico, a urbanização e o aumento dos padrões de consumo apontam para crescimento na quantidade e complexidade dos RSU - como subprodutos inevitáveis da atividade humana - , favorecendo graves problemas sanitários, principalmente nos países em desenvolvimento. Neste contexto, constata-se que os avanços do consumo e da industrialização, adicionados à integração de pequenas comunidades aos mercados, indicam aumento na geração de RSU em todo o mundo.

Para a realidade brasileira, a geração de RSU também tem registrado considerável elevação, incrementada pela crescente urbanização, que atingiu 85\% da população total do País (IBGE, 2011). Tal incremento tem se manifestado em todas as regiões geográficas, principalmente nas cidades com maior concentração populacional, cujas médias de geração superam 1,2 kg/hab.dia. A quantidade é equivalente à verificada nos países desenvolvidos e revela hábitos de consumo e descarte dos moradores dessas cidades, que ainda não refletiram políticas implementadas para reduzir o volume de resíduos (ABRELPE, 2010).

O fenômeno de urbanização provocou, ao longo do século XX, pressão significativa em diversos setores da economia nacional, em especial nos serviços de distribuição de energia elétrica, abastecimento de água, esgotamento sanitário e coleta de RSU. Aliado a isso, com a estabilidade econômica iniciada a partir de 1994, o País passou a apresentar crescimento do Produto Interno Bruto que resultou em aumento da renda de parcelas significativas da população. A nova distribuição socioeconômica representou elevações da demanda por diversos produtos que passaram a ser consumidos em diferentes escalas, favorecendo a geração dos resíduos aos mesmos associados.

Em consonância com tal tendência, a prestação do serviço de limpeza urbana apresentou, em 2010, crescimento de 8\% comparado a 2008. Dentre as localidades pesquisadas, a Região Sudeste respondeu por $53 \%$ do total de resíduos coletados, contra 22, 11, 8 e 6\% respectivamente nas regiões Nordeste, Sul, Centro-Oeste e Norte. Quando se observa o processo de coleta seletiva, diretamente vinculado à reciclagem e ao reaproveitamento, novamente a Região
Sudeste se destaca, com 78,7\% dos municípios contemplando iniciativas desta natureza, enquanto as regiões Sul, Norte, Nordeste e Centro-Oeste totalizam 76,2, 44,1, 34,2 e 26,1\%, respectivamente (ABRELPE, 2010).

\section{Aspectos teóricos de modelagem e algumas aplicações em resíduos sólidos}

A necessidade de se compreender o mundo em que se vive incentiva o ser humano a estabelecer relações entre elementos, com o intuito de prover maior controle do meio no qual as populações estão inseridas. Surge a necessidade de se conceber modelos que consistem na forma pela qual se obtém observação estruturada da realidade. Dentre diversos aspectos técnicos do problema a ser modelado, torna-se imprescindível analisar três dimensões que interferem na complexidade da modelagem. O meio ambiente da problemática (tratável ou intratável), o domínio das variáveis (poucas e homogêneas ou muitas e heterogêneas) e a dinâmica do fenômeno (determinístico, estocástico ou indeterminado) constituem dimensões que indicam se a modelagem tornar-se-á factível ou não. Desta forma, o desenvolvimento de um modelo deve preservar os seguintes passos: definição do problema, formulação e construção do modelo inicial, validação do modelo, simulação do modelo, reformulação do modelo e aplicação do modelo (GOLDBARG \& LUNA, 2000).

Já sob o ponto de vista de Altiok e Melamed (2007), os modelos são representações simplificadas de um sistema complexo visando a prover projeções de medidas de interesse, podendo apresentar variações em suas formas físicas, matemáticas, analíticas e computacionais. Os mesmos autores defendem que as modelagens devem ser elaboradas fazendo-se uso da seguinte sequência de processos: análise do problema, coleta dos dados, construção do modelo, verificação do modelo, validação do modelo, projeção e condução de experimentos de simulação, análise dos resultados e recomendações finais. Apesar de claramente identificadas, as fases apresentam riscos vinculados à experiência do analista, assim como ao conhecimento específico do problema a ser simulado. Tais fatores devem ser observados a fim de se garantir a confiabilidade desejada, representando a compatibilidade com o mundo real.

Estudo realizado por Solano (1999) indicou modelagem matemática para representar fluxos de massa em processo de gerenciamento municipal de RSU. A metodologia proposta baseou-se em programação linear, na qual duas funções objetivo compostas buscaram minimizar os custos de manejo dos resíduos, assim como o custo de emissões de gases nocivos ao meio ambiente. Restrições tecnológicas foram representadas por equações restritivas de ordem linear e validações realizadas por combinações de cenários estratégicos baseados em uma região urbana hipotética de médio porte. Todavia, permaneceu a lacuna relacionada ao comportamento dos volumes totais de resíduos gerados e suas projeções. 
Em linha de investigação semelhante, Bach et al. (2004) delinearam um estudo estabelecendo a relação dos fatores sociodemográficos e logísticos com a geração de resíduos de papel. Nesse trabalho, evidenciaram-se os principais componentes que apresentaram afinidade com o aumento na coleta de resíduos de papel, com o intuito de subsidiar tomadores de decisão e apoiar planos estratégicos voltados à área específica em questão. Os dados amostrais possibilitaram estabelecer correlações entre o aumento de resíduos de papel e fatores comportamentais como turismo e viagens de negócio, poder aquisitivo da população e o número de empregados de empresas, indústrias e serviços.

Custos econômicos e ambientais associados foram considerados em estudo realizado com objetivo de desenvolver um modelo para gerenciar o manejo de RSU na cidade de Mumbai, região metropolitana mais populosa da Índia. Mais uma vez, optou-se pela modelagem por meio de programação linear para tratamento do problema, porém, utilizando-se dados de questionários aplicados, uma vez que a municipalidade não dispunha de informações sistematizadas (RATHI, 2007).

Aplicações das práticas de modelagem foram empregadas num estudo de simulação da produção de resíduos sólidos com a finalidade de geração de energia (PANAGIOTIDIOY; GEKAS; STAVRAKAKIS, 2009). Tal abordagem foi desenvolvida para três distritos gregos, onde o balizamento dos trabalhos também pautou-se em dados apresentados pelo serviço nacional de estatística daquele País.

Em nível nacional, a heterogeneidade da economia brasileira resulta em diversos padrões para a geração de RSU. A partir de uma amostragem representativa em cidades brasileiras, a Associação Brasileira de Empresas de Limpeza Pública e Resíduos Especiais (ABRELPE, 2010) elaborou um relatório a fim de retratar o panorama da geração e reciclagem dos RSU nos municípios pesquisados. Análises mostraram alta correlação entre a magnitude da população das cidades e a produção per capita de resíduos gerados. Em todas as regiões geográficas do Brasil, tal coeficiente de determinação $\left(\mathrm{R}^{2}\right)$ suplantou 0,80. Todavia, o trabalho não estratificou as populações em classes socioeconômicas, e tampouco estabeleceu modelos preditivos para a geração de resíduos domiciliares nos ambientes urbanos.

$\mathrm{Na}$ proposta de modelagem desenvolvida por Caldeira (2008), objetivou-se elaborar um modelo estatisticamente significativo que correlacione variáveis geográficas, demográficas, políticas e socioeconômicas à cobertura do serviço público de coleta dos resíduos sólidos em domicílios de municípios mineiros. Na oportunidade, modelaram-se hierarquicamente os determinantes, tendo-se como variável resposta a presença de coleta dos RSU nos domicílios urbanos de Minas Geais. O trabalho apresenta ainda vasta abordagem em análises descritivas, porém permaneceu novamente a lacuna relacionada ao interesse voltado à ótica do planejamento de futuras demandas de serviços de coleta e tratamento de resíduos.
Corroborando a iniciativa de se pesquisar modelos de previsão de geração de RSU, Silva (2008) buscou elucidar algumas variáveis que pudessem aferir a produção de resíduos em Belo Horizonte. Mais especificamente, a abordagem baseou-se na investigação dos diferenciais socioeconômicos e demográficos e suas influências na composição e geração de RSU no Município. Na ocasião, foram cruzados dados advindos da Superintendência de Limpeza Urbana (órgão ligado à Prefeitura Municipal) com as informações provenientes do Censo Demográfico de 2000, perfazendo-se análises multivariadas em clusters espaciais por meio do software Stata, tanto sob o prisma demográfico populacional, quanto sob a ótica socioeconômica. Os resultados encontrados confirmaram correlação significativa entre renda, escolaridade e geração de resíduos sólidos. No entanto, pelo próprio propósito da pesquisa, discutiram-se apenas aspectos voltados a grupos de faixas etárias, número de filhos por casais, além de perfis urbano-contemporâneos dos chefes de domicílio.

Contudo, a despeito da relevância dessa pesquisa em se evidenciar a renda e a educação como fatores de maior interveniência na produção resíduos sólidos — respondendo por 65\% da variância —, sob o ponto de vista e interesse da engenharia, não foi apresentado modelo matemático que permitisse estimar ou projetar a geração de RSU, visando ao planejamento sanitário das cidades sob cenários hipotéticos futuros.

Diante do referencial teórico pesquisado, a principal motivação deste trabalho ocorre no momento no qual a população brasileira começa a sofrer modificações em sua estrutura socioeconômica, especificamente a partir do ano 2000. Programas de distribuição de renda do Governo Federal têm fomentado alterações significativas nos rendimentos per capita dos consumidores. Diante dessa realidade, surge a preocupação em garantir o atendimento das futuras demandas. Para tanto, torna-se apropriado estimar as necessidades da sociedade de acordo com cenários econômicos projetados. Neste cenário, decidiu-se aplicar modelagem utilizando-se a técnica de Data Mining (técnica de mineração de dados na qual se coletam massas de dados para posterior análise e tratamento) a qual tem como axioma o desenvolvimento de modelos que mostrem resultados baseados apenas na representatividade que seus próprios dados refletem.

Diante do exposto, a pesquisa versa sobre a elaboração de modelo para estimativa da geração de resíduos sólidos pelas populações dos centros urbanos.

\section{Metodologia}

A metodologia implementada emprega o cruzamento de microdados de pesquisas socioeconômicas conjunturais do Instituto Brasileiro de Geografia e Estatística (IBGE) aos dados de coleta de RSU de microrregiões específicas dentro de um município escolhido — em primeira instância, Belo Horizonte. O tratamento dos dados socioeconômicos 
consistiu, a partir do arquivo mensal de microdados do IBGE, em proceder à entrada das informações ao software Statistical Package for the Social Sciences (SPSS), empregando uma rotina desenvolvida para tal. Assim, foram lidas do arquivo original de microdados apenas as variáveis pertinentes ao trabalho, procedendo-se à seleção, agregação dos dados de interesse e ao cálculo dos indicadores para cada região abordada. A obtenção dos dados e dos indicadores socioeconômicos realizou-se por meio da pesquisa conjuntural, denominada Pesquisa Mensal do Emprego (PME), na qual se afere de forma mensal a evolução do emprego e renda nas principais regiões metropolitanas. O tamanho da amostra da PME, especificamente em Belo Horizonte, contemplou 3,1 mil domicílios com, aproximadamente, 10,2 mil moradores abarcados mensalmente pela pesquisa (IBGE, 2010).

Os indicadores socioeconômicos foram, na sequência, agregados segundo as regiões administrativas da Prefeitura Municipal de Belo Horizonte, conforme mostra a Figura 1. No caso específico, o modelo de projeção de geração de RSU baseou-se na renda, na classificação, no tamanho e na distribuição econômica da população estudada. Para cada classe econômica determinou-se a produção de resíduos per capita típica. Funções foram ajustadas por meio de regressões e agrupamentos destacados segundo os rendimentos per capita com propósito de se representar a distribuição das produções

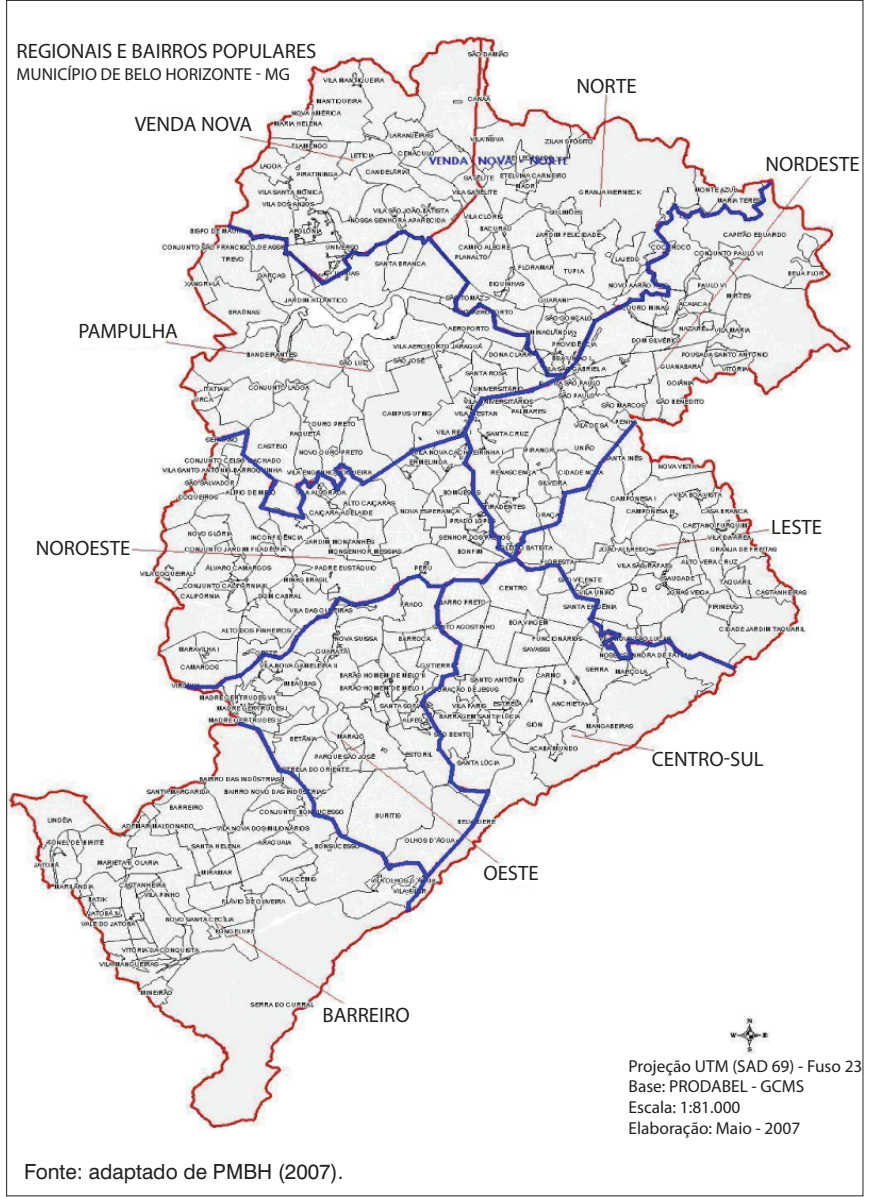

Figura 1 - Município de Belo Horizonte e a compatibilização espacial entre agências operacionais e regionais abordadas nesta pesquisa. entre as diversas classes econômicas. Parte da metodologia utilizada nesta pesquisa balizou-se no trabalho no qual se fez a aplicação de microdados de pesquisas conjunturais na análise de sistemas de abastecimento de água (DIAS; MARTINEZ; LIBÂNIO, 2010).

Os dados históricos de coleta de RSU foram obtidos junto à Superintendência de Limpeza Urbana (SLU, 2011) para Belo Horizonte, e ao Departamento Municipal de Limpeza Urbana (DMLU, 2011) para Porto Alegre. Foram utilizados dados mensais de coleta agrupados por regionais administrativas referentes ao período de janeiro de 2006 a dezembro de 2010, totalizando 540 dados de geração per capita de resíduos. Ressalta-se que os dados relativos às massas de resíduos coletados consideram as coletas domiciliares, ou seja, os resíduos gerados por edificações residenciais, comerciais, públicas e de prestação de serviços. Não foi objeto desta pesquisa a massa de resíduos lançados em via pública, de varrição, especiais ou de saúde. A principal forma de coleta no âmbito domiciliar abordada por este trabalho deu-se por meio de caminhões compactadores, além de basculantes, ou caçambas estacionárias para os casos de vilas e favelas de difícil acesso viário. Consideraram-se ainda a coleta seletiva de papel, metal, plástico, vidro e resíduos orgânicos.

Em relação à especificação das variáveis, procedeu-se às comparações na ordem apresentada na Tabela 1, objetivando melhor estruturar o acompanhamento das análises por meio das diversas confrontações de dados e alcançar a correlação mais significativa.

As rendas deflacionadas levaram em conta a evolução da inflação refletida pelo Índice Nacional de Preços ao Consumidor Amplo (INPC-A) do IBGE ao longo do período observado, no sentido de compatibilizar a evolução das rendas ao real poder de compra dos consumidores. Em cada regional administrativa, os rendimentos médios foram deflacionados mensalmente segundo os índices oficialmente divulgados. Desta forma, o termo "renda deflacionada" vincula-se ao valor auferido pela população, já descontados os aumentos de preços, acumulados desde o período inicial do histórico de dados.

\section{Resultados e Discussão}

\section{Proposição do modelo}

Utilizando-se dados da SLU (2011) verificou-se nítida evolução da produção de RSU em Belo Horizonte. O acréscimo registrado no período analisado de pouco menos de cinco anos foi da ordem de $23 \%$, atingindo máximo mensal de aproximadamente 55 mil toneladas (março de 2010). Evidenciou-se também grande variabilidade dos resultados mensais, mesmo entre meses consecutivos. Vale

Tabela 1 - Regressões realizadas para elaboração do modelo.

$\begin{array}{lc}\text { Variável explicativa } & \text { Variável dependente } \\ \text { Renda per capita deflacionada } & \text { Geração domiciliar per capita } \\ \text { Renda per capita deflacionada } & \text { Geração domiciliar total }\end{array}$


salientar que os valores se referem às massas coletadas pela SLU e suas concessionárias, sendo obviamente inferiores ao total produzido, já que parte não é coletada e resta, portanto, sem ser computada. $\mathrm{O}$ aumento das quantidades produzidas pode, em parte, ser creditado à melhora nos serviços de coleta, e não somente à maior produção em si. Otimização de percursos, terceirização dos serviços, coletas noturnas, equipamentos mais modernos e estratégias que contam com maior participação da população são algumas das variáveis que podem ter permitido esta melhora nos resultados sem que a quantidade propriamente dita seja alterada.

Adicionalmente, fração dos RSU municipais produzidos vem sendo reciclada, oficialmente ou não, o que também mascara resultados. Em 2009, estima-se que a SLU tenha reciclado 5,2\% dos RSU coletados, sendo a maior parte constituída por resíduos da construção civil, que não fazem parte deste estudo. Mantendo mesma tendência, a reciclagem elevou-se para aproximadamente 10\% do total coletado, e também a maior fração se deveu aos entulhos. Paradoxalmente, o relatório de 2010 da SLU reporta sensível diminuição da produção de RSU na cidade em relação a 2009, quando os indicadores econômicos, espelhando o momento de vigor por que passa a economia brasileira, se mostravam altamente positivos, o que levaria à maior produção de RSU de todos os tipos.

Quando se parte para uma análise adimensional, considera-se cada ponto dividindo-se o valor nominal pelo valor médio observado. Assim, a amplitude dos picos de geração de RSU, máximos e mínimos, também pôde ser avaliada. A geração de RSU média passa a ser representada pelo valor adimensional 1 , e os picos de máximos e mínimos mostram o percentual de variação em relação a essa média. A observação da Figura 2 permite afirmar que os picos manifestaram tendência de aumento de suas amplitudes, uma vez que os máximos são aproximadamente de 10 a 20\% superiores à média do período, e os picos de mínimos encontram-se, aproximadamente, 10 a $15 \%$ inferiores ao valor médio do período.

Uma característica evidenciada na Figura 2 é a influência sazonal na geração domiciliar de RSU. Pode-se afirmar que há influência aparente na oscilação da produção causada por questões meramente associadas aos meses do ano. Neste caso, além de influências climáticas e meteorológicas, pode-se considerar o final do ano, quando o $13^{\circ}$ salário injeta bilhões de reais na economia, o que favorece o aumento da geração de RSU.

A partir da evolução populacional, possibilitou-se proceder ao cálculo da geração mensal per capita domiciliar de resíduos, calculada para cada regional administrativa de Prefeitura Municipal de Belo Horizonte e seus bairros correspondentes. Dessa análise, depreende-se que as oscilações ocorrem de forma similar em todas as séries de dados. Esse comportamento sugere que os efeitos causadores das referidas oscilações são intervenientes, da mesma maneira, em todas as áreas de Belo Horizonte, não existindo fatores sazonais com diferenciações entre as regiões da capital, conforme se evidencia na Figura 3.
No entanto, observam-se variações nos valores da produção de resíduos sólidos das regiões da cidade, seguramente associadas a suas rendas per capita médias e a outras variáveis (tipos de atividades predominantes, tipos de usuário/morador, etc). Destaca-se a Regional Centro-Sul, com médias bastante superiores às demais regionais, que além de constituir a parte histórica da cidade, mais adensada e verticalizada, concentra parte expressiva do comércio e de serviços.

Dando sequência ao processo analítico do comportamento temporal das variáveis, passou-se a considerar o poder de compra da população das nove regionais administrativas. Desta forma, os rendimentos per capita reais foram ajustados para que refletissem o seu real valor em relação à evolução do índice oficial de inflação, ou seja, foram deflacionados de acordo com a inflação verificada pelo INPC-A divulgado pelo $\mathrm{IBGE}_{(\mathrm{b})}$ (2011). A discrepância entre a Regional Centro-Sul manifestou-se em mesma magnitude. No período analisado, nas camadas socioeconômicas inferiores, como as presentes nas regionais Barreiro, Venda Nova/Norte e Noroeste, registraram-se ganhos relativos nos rendimentos em torno de 30 a 40\%, considerando-se a deflação aplicada. Na Regional Centro-Sul, mais favorecida

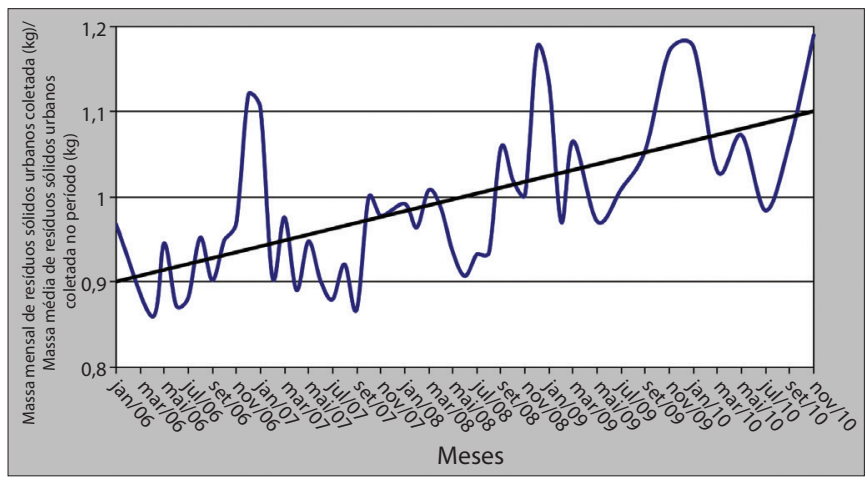

Figura 2 - Evolução histórica da geração domiciliar de resíduos sólidos em Belo Horizonte em valores adimensionalizados.

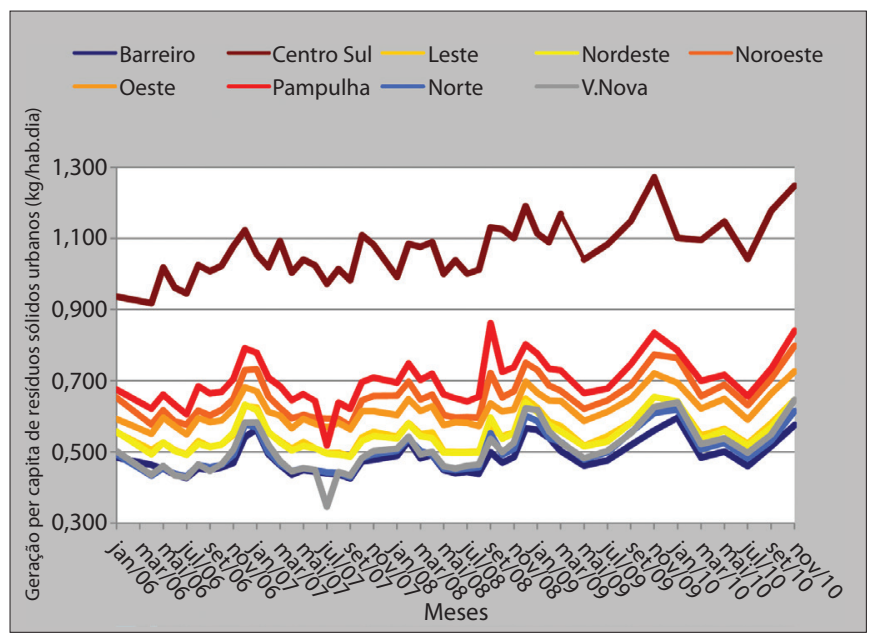

Figura 3 - Evolução da geração domiciliar per capita de resíduos sólidos urbanos, segundo cada regional administrativa da Prefeitura Municipal de Belo Horizonte. 
economicamente, os ganhos relativos de rendimento per capita, em torno de 33\%, manifestaram-se somente depois de 2008. Já no caso de regionais intermediárias, como Leste e Nordeste, os ganhos foram bem mais modestos ou até mesmo inexpressivos, como no caso da Regional Oeste.

Posteriormente às regressões realizadas, estabeleceu-se a função que melhor aderência apresentou à dispersão dos dados amostrais, cujo coeficiente de determinação ( $\left.\mathrm{R}^{2}\right)$ foi 0,8525 (Figura 3).

Depreende-se do gráfico apresentado pela Figura 4 que, em termos generalizados, a produção domiciliar de resíduos sólidos em Belo Horizonte pode ser representada pela Equação 1.

$Y=-0,00000005 x^{2}+0,0006 x+0,2848$

Na qual:

$Y=$ Produção diária de resíduo sólido per capita (kg/hab.dia);

$x=$ Renda per capita mensal (R $\$ / m e ̂ s)$.

Assim, quando se aplica a Equação 1 para cada estrato socioeconômico estipulado, descortina-se o modelo de estimativa de geração total de resíduos sólidos de Belo Horizonte a partir da Equação 2.

$C=\sum_{i}^{n} P *\left(-0,00000005 x^{2}+0,0006 x+0,2848\right)$

Na qual:

$C=$ Quantitativo total produzido de resíduo sólido domiciliar por dia (kg/dia);

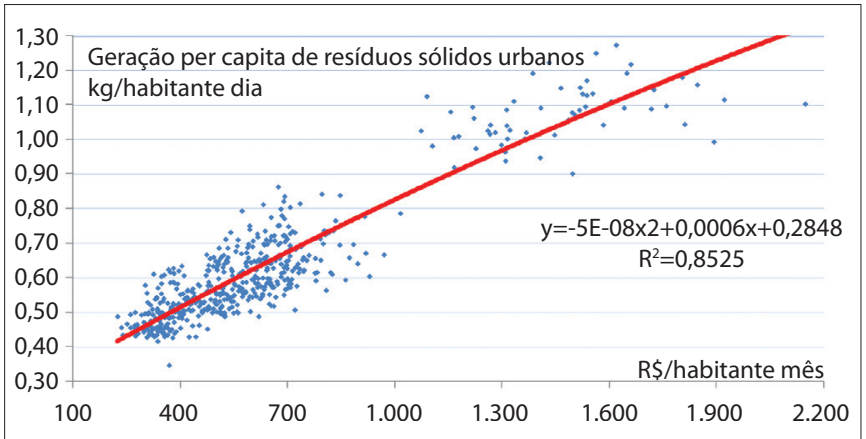

Figura 4 - Renda per capita versus geração per capita de resíduos sólidos urbanos no município de Belo Horizonte. $x=$ Renda per capita mensal média de cada estrato socioeconômico arbitrado (R\$/mês);

$P=$ População existente em cada estrato socioeconômico arbitrado na região considerada;

$i=$ Quantidade de faixas socioeconômicas arbitradas, variando de 1 a $n$

Portanto, pode-se calcular a parcela da geração de RSU referente a cada da classe socioeconômica, atribuindo-se a cada uma o peso relativo decorrente do total de habitantes a ela pertencente.

\section{Validação do modelo}

Com objetivo de avaliar o modelo e a consistência da metodologia desenvolvidos, procedeu-se à comparação entre os quantitativos de resíduos sólidos domiciliares informados pela SLU (2011) e pelo DMLU (2011), e os quantitativos calculados por meio da Equação 2. A validação do modelo foi testada, portanto, a partir da comparação entre a geração total obtida matematicamente pelo modelo e a massa física de resíduos efetivamente coletada e informada pelas prestadoras do serviço.

Desta forma, multiplicando-se os habitantes existentes em cada classe social pela renda média per capita obtida para as mesmas classes, afere-se a parcela de produção de resíduos referente a cada classe socioeconômica dos municípios abordados. No que tange à distribuição atual das populações, segundo cada classe econômica, adotaram-se informações da ABEP (2010) sintetizadas na Tabela 2. Ressalta-se que esses últimos indicadores foram divulgados em 2010, estando referenciados de forma compatível ao período comparativo dos demais dados.

Em Belo Horizonte, a população recenseada durante o Censo Demográfico de 2010 atingiu 2.375.151 habitantes, sendo que, no tocante ao número médio de moradores por domicílio, obteve-se 3,1. Analogamente, a população de Porto Alegre atingiu 1.409.939 habitantes em 2010, com número médio de moradores por domicílio de 2,8 (IBGE, 2011). Tais valores foram empregados para a estimativa da renda per capita para cada classe econômica, a partir da renda familiar apresentada na Tabela 2.

Por meio do emprego da Equação 2 para as duas cidades, estimou-se a geração de resíduos sólidos, por classe socioeconômica, conforme a Tabela 3.

Tabela 2 - Populações dos municípios e regiões metropolitanas brasileiros segundo classes socioeconômicas.

\begin{tabular}{lcccc} 
Classe econômica & $\begin{array}{c}\text { Renda familiar mensal } \\
(\mathrm{R} \$ / \text { mês) }\end{array}$ & Belo Horizonte (\%) & Distribuição da população \\
Porto Alegre (\%) & 0,2 & Brasil (\%) \\
A1 & $14.366,00$ & 0,6 & 4,9 & 4,6 \\
A2 & $8.099,00$ & 3,2 & 11,3 & 9,1 \\
B1 & $4.558,00$ & 7,7 & 22,9 & 18,0 \\
B2 & $2.327,00$ & 16,1 & 27,0 & 24,5 \\
C1 & $1.391,00$ & 24,2 & 21,0 & 23,9 \\
C2 & 933,00 & 23,8 & 11,9 & 17,9 \\
D & 618,00 & 23,0 & 0,8 & 1,6 \\
E & 403,00 & 1,4 & & \\
\hline
\end{tabular}


A fim de avaliar o resultado do modelo sintetizado pela Equação 2 e demonstrado na Tabela 3, procedeu-se à comparação do mesmo com o histórico de geração de resíduos coletados em Belo Horizonte e Porto Alegre. Para a primeira, no período estudado foi coletada média mensal de 44.705,26 toneladas (SLU, 2011), superior em 5,2\% à massa estimada pelo modelo. Em contrapartida, no mesmo período, em Porto Alegre, a massa média coletada atingiu 29.001,68 toneladas, 3,3\% inferior ao estimado pelo modelo.

Considerando-se as estimativas obtidas para projeção da geração de resíduos sólidos em Belo Horizonte e Porto Alegre, faz-se possível tecer algumas suposições. A primeira refere-se ao poder de compra observado nas duas capitais estudadas, o que possivelmente pode distinguir a geração de resíduos em cada família. De acordo com a Tabela 2, Belo Horizonte apresenta $27,6 \%$ da sua população pertencente às classes socioeconômicas iguais ou superiores à B2, enquanto em Porto Alegre esta parcela atinge 36,3\% da população.

Dessa perspectiva emergem duas suposições quase contraditórias. A primeira vincula-se à geração média per capita de resíduos sólidos de 0,627 kg/hab.dia em Belo Horizonte, enquanto em Porto Alegre esta geração é 9,4\% superior $(0,686 \mathrm{~kg} / \mathrm{hab}$.dia). A segunda suposição prende-se ao emprego de tecnologias mais modernas que, por apresentarem alternativas de reaproveitamento de resíduos, podem incentivar as famílias de maior renda à redução na geração. Como consequência dos estratos socioeconômicos de maior renda verificados, Porto Alegre tem maior tradição na coleta seletiva e, ao se avaliar a produção de resíduos, tem-se que computar os valores reciclados.

Tabela 3 - Estimativa da geração de resíduos sólidos por classe socioeconômica para Belo Horizonte e Porto Alegre.

\begin{tabular}{lrr} 
Classe econômica & \multicolumn{2}{c}{ Geração de resíduos sólidos $\left(\mathrm{kg}\right.$. dia $^{-1}$ ) } \\
\cline { 2 - 3 } A1 & Belo Horizonte & Porto Alegre \\
\hline A2 & $28.380,90$ & $5.772,66$ \\
B1 & $114.859,63$ & $139.597,19$ \\
B2 & $193.632,53$ & $179.888,54$ \\
\hline C1 & $270.432,55$ & $241.792,78$ \\
C2 & $312.778,85$ & $217.237,15$ \\
D & $260.523,33$ & $141.842,12$ \\
E & $219.726,73$ & $69.622,89$ \\
\hline Total mensal (t/mês) & $12.035,73$ & $4.175,44$ \\
\hline
\end{tabular}

Vale ressaltar que há incerteza com relação aos registros da massa de resíduos coletados, pois esses são obtidos a partir do número de caminhões transportados e pesados antes de sua efetiva descarga. Assim, estima-se erro de medição da ordem de 5\%. A tal incerteza, soma-se à massa desviada do fluxo principal de geração dos RSU, via reciclagem, reutilização ou compostagem da fração orgânica.

Por fim, a despeito de tais limitações, sob o ponto de vista de futuros cenários de alterações na estrutura de distribuição socioeconômica das populações e de ter sido elaborado calcado nos dados de Belo Horizonte, o modelo abre a perspectiva de subsidiar políticas ou de orientar diretrizes voltadas ao planejamento de serviços de coleta e de destinação final de resíduos sólidos.

\section{Conclusão e Recomendações}

As análises dos resultados obtidos pelo modelo evidenciaram que as principais variáveis explicativas foram a renda e a dimensão de cada classe econômica das populações, sendo que os demais fatores intervenientes representaram baixo impacto e menor reflexo nas demandas domiciliares do insumo em questão (RSU). Tendo em vista o significativo grau de correlação, o trabalho permite afirmar que o modelo delineado poderá contribuir para estudos e projeções de geração de resíduos a outras regiões metropolitanas brasileiras.

Recomenda-se a continuidade da obtenção dos dados históricos para se aumentar o horizonte amostral, como também a utilização da metodologia em outras regiões para que, no futuro, resultados mais precisos e conclusivos possam ser obtidos para diversas regiões e cenários econômicos brasileiros.

\section{Agradecimentos}

Os autores manifestam seus agradecimentos à Superintendência de Limpeza Urbana da Prefeitura Municipal de Belo Horizonte, ao Departamento Municipal de Limpeza Urbana da Prefeitura Municipal de Porto Alegre, à Fundação de Amparo à Pesquisa do estado de Minas Gerais e ao Instituto Brasileiro de Geografia e Estatística pelo apoio institucional dispensado para a realização deste trabalho.

\section{Referências}

ALTIOK, T. \& MELAMED, B. (2007) Simulation modeling and analysis with arena. New Jersey, USA. Elsevier Science, $440 \mathrm{p}$

Associação Brasileira de Empresas de Limpeza Pública e Resíduos Especiais - ABRELPE. (2010) Panorama do resíduos sólidos no Brasil 2009. São Paulo. ABRELPE/ISWA, 210 p.

Associação Brasileira de Empresas de Pesquisa - ABEP. Critério de Classificação Econômica Brasil. São Paulo, SP. < http://www.abep.
org/novo/Content.aspx?SectionID=84 > Acesso em: 25 nov. 2010.

Associação Brasileira de Normas Técnicas - ABNT. (2004) Resíduos Sólidos - Classificação, NBR 10004. Rio de Janeiro, 71 p.

BACH, H.; MILD, A.; NATTER, M.; WEBER, A. (2004) Combining socio-demographic and logistic factors to explain the generation and collection of waste paper. Resources, Conservation and Recycling, v. 41, n. 1, p. 65-73. 
BRASIL. Instituto Brasileiro de Geografia e Estatística - IBGE. (2008) Pesquisa Mensal do Emprego. <http://www.ibge.gov.br/home/ estatistica/indicadores/trabalhoerendimento/pme_nova/default.shtm > Acesso em: 6 ago. 2010.

BRASIL. Instituto Brasileiro de Geografia e Estatística - IBGE. (2010) Cidades. <http://www.ibge.gov.br/cidadesat/topwindow.htm?1> Acesso em: 2 maio 2011

BRASIL. Instituto Brasileiro de Geografia e Estatística - IBGE. (2010) Índice Nacional de Preços ao Consumidor Amplo - IPCA e Índice Nacional de Preços ao Consumidor - INPC. <http://www.ibge.gov.br/ home/estatistica/indicadores/precos/inpc_ipca/defaultinpc.shtm> Acesso em: 17 out. 2011.

CALDEIRA, M.M. (2008) Estudo dos determinantes da coleta domiciliar e da disposição final dos resíduos sólidos urbanos em Minas Gerais. 2008. Dissertação (Mestrado em Saneamento, Meio Ambiente e Recursos Hídricos) - Universidade Federal de Minas Gerais, Belo Horizonte.

DIAS, D.M.; MARTINEZ, C.B.; LIBÂNIO, M. (2010) Avaliação do impacto da variação da renda no consumo domiciliar de água. Revista Engenharia Sanitária e Ambiental, v. 15, n. 2, p. 155-166.

GOLDBARG, M. \& LUNA, H. (2000) Otimização combinatória e programação linear modelos e algoritmos. Rio de Janeiro. Editora Campus, $649 \mathrm{p}$.

PANAGIOTIDIOY, N.; GEKAS, V.; STAVRAKAKIS, G. Modeling and evaluation of electrical energy recovery from urban solid waste: the case of Chania-Crete-Grecce. Proccedings of the $11^{\text {th }}$ International Conference on Environmental Science and Technology. Technical University of Crete, Grecce, 2009

PREFEITURA MUNICIPAL DE BELO HORIZONTE. (2007) Regionais e Bairros Populares. Belo Horizonte, Prodabel.

PREFEITURA MUNICIPAL DE BELO HORIZONTE. (2011) Superintendência de Limpeza Urbana - SLU. (2011) Relatório Anual de Atividade de Limpeza Urbana (2007 a 2010). Belo Horizonte, Prefeitura Municipal de Belo Horizonte.

PREFEITURA MUNICIPAL DE PORTO ALEGRE. Departamento Municipal de Limpeza Urbana - DMLU. (2010) Quantitativo de resíduos destinados às unidades gerenciadas pela divisão de destino final. Disponível em: http://lproweb.procempa.com.br/ pmpa/prefpoa/dmlu/usu_doc/dadosdmlu042011.pdf. Acesso em: 23 nov. 2011

RATHI, S. (2007) Optimization model for integrated municipal solid waste management in Mumbai, India. Environment and Development Economics. United Kingdom, Cambridge University Press.

SILVA, H. (2008) Aspectos demográficos associados à geração de resíduos domiciliares no município de Belo Horizonte, 2002. 203 p. Dissertação (Mestrado em Demografia) - Universidade Federal de Minas Gerais, Belo Horizonte.

SOLANO, E. (1999) Integrated solid waste management alternatives in consideration of economic and environmental factors: a mathematical model development and evaluation. 105 p. Tese (Ph.D em Filosofia) North Carolina State University, Raleigh, USA. 